\title{
Mucinous adenocarcinoma of ovary
}

INSERM

\section{Source}

INSERM. (1999). Orphanet: an online rare disease and orphan drug data base. Mucinous adenocarcinoma of ovary. ORPHA:398961

Mucinous adenocarcinoma of ovary is a rare, malignant epithelial tumor of the ovary characterized, macroscopically, by a large, usually unilateral tumor with smooth surface and evenly distributed cystic and solid areas and, histologically, by a complex papillary growth pattern with microscopic cystic glands and necrotic debris. Patients often present with pelvic pain and pressure, abdominal mass or gastrointestinal problems such as early satiety or bloating. 